\title{
Modeling the THF clathrate hydrate dynamics by combining molecular dynamics and quasi-elastic neutron scattering
}

\author{
Eric Pefoute ${ }^{\mathrm{a}, \mathrm{b}}$, Ludovic Martin-Gondre ${ }^{\mathrm{a}, \mathrm{c}, 1}$, Jacques Ollivier ${ }^{\mathrm{d}}$, Jean-Christophe Soetens ${ }^{\mathrm{a}}$, \\ Margarita Russina ${ }^{\mathrm{b}}$, Arnaud Desmedt ${ }^{\mathrm{a}, *}$ \\ a Institut des Sciences Moléculaires, UMR5255 CNRS - Université de Bordeaux, Talence, France \\ ${ }^{\mathrm{b}}$ Helmholtz Zentrum Berlin, Berlin, Germany \\ cInsitut UTINAM - Univ. Bourgogne Franche-Comté, CNRS UMR 6213, Besançon, France \\ ${ }^{\mathrm{d}}$ Institut Laue Langevin, Grenoble, France
}

\begin{abstract}
The dynamics of the THF molecule encapsulated in the type II clathrate hydrate matches the MD-QENS observation time (typically $0.1-10 \mathrm{ps}$ ) between $100 \mathrm{~K}$ and $270 \mathrm{~K}$. Spatial and time characteristics of the THF molecule's dynamics obtained by means of MD simulations are in agreement with those experimentally determined by means of quasielastic neutron scattering. A detailed model of the THF dynamics is then proposed through the calculations of MD-derived properties. Reorientational relaxation has been observed on a timescale of $0.7 \pm 0.1 \mathrm{ps}$ at $270 \mathrm{~K}$ with activation energy of $3.0 \pm 0.3 \mathrm{~kJ} / \mathrm{mol}$ in addition to a highly damped rotational excitation occurring in the plane of the THF molecule with a period of $c a$. 2 ps. Moreover, the anisotropic cage energy landscape of the THF clathrate hydrate is revealed through a comprehensive investigation of THF orientational distribution functions, revealing the occurrence of preferred orientation of the THF molecule within the cage.
\end{abstract}

\section{Introduction}

Clathrates hydrates consist in a crystalline water network forming cages encapsulating foreign molecules [1]. The natural existence of large quantities of hydrocarbon hydrates in deep oceans and permafrost is probably at the origin of numerous applications in the broad areas of energy and environmental sciences [2-4]. At a fundamental level, extensive studies have been done on their thermodynamics and equilibrium properties $[2,3]$ and numerous studies are devoted to the formation, inhibition and decomposition of gas hydrates [5], to their anomalous thermal conductivities [6] or to the properties of ionic clathrate hydrates [7].

The tetrahydrofuran (THF) molecule is probably one of the most widely used guest molecule to form clathrate hydrate. It is used as promoter for forming gas hydrates since it permits relaxing the formation condition (e.g. decreased of the formation pressure). The solubility of THF within water and the formation conditions closed to room temperature ( $277 \mathrm{~K}$ at 1 bar) provide clear advantages compared with other gas hydrates, making the THF clathrate hydrate commonly studied and used as analogue hydrate. THF

\footnotetext{
* Corresponding author.

E-mail address: arnaud.desmedt@u-bordeaux.fr (A. Desmedt).

1 Present address: UTINAM, France.
}

hydrate is commonly studied as a substitute for natural gas hydrates [8-11], used as an additive to reduce the formation pressures for flow assurance [1], hydrogen storage [12,13], gas separation schemes $[14,15]$ or refrigeration applications [16]. There have been numerous fundamental studies on the THF clathrate hydrate. The thermodynamics properties have been the subject of various investigations going from the precise determination of its pressure-temperature phase diagram [17-19] to the formation kinetics and mechanisms [20-23]. The vibrational signatures of the THF clathrate hydrate have been worked over by means of Raman scattering [24-28] and IR absorption [29,30]. By means of X-ray [31], and neutron diffraction $[32,33]$, it has been showed that the THF clathrate hydrate crystallizes in the type II structure with only the large cages filled with THF molecules (leading to the stoichiometric compound THF- $17 \mathrm{H}_{2} \mathrm{O}$ ). Moreover, the two hydrogen atoms of the water molecules occupied four equiprobable positions around each oxygen atom. This disorder of the H-bond network in the water substructure has been revealed by means of dielectric spectroscopy [34-36], of ${ }^{1} \mathrm{H}$ NMR [37] and of solid state ${ }^{2} \mathrm{H}$ NMR [38-42]. The timescale of the water dynamics ranges from the microsecond to the millisecond above $193 \mathrm{~K}$. The impact of acidic additives - known to increase host proton mobility in the host substructure [43-45] - has been investigated on its thermodynamical, vibrational and structural properties [46]. Finally, the THF 
dynamics has been explored by means of dielectric spectroscopy [34], of ${ }^{1} \mathrm{H}$ NMR [37,47-49], of ${ }^{2} \mathrm{H}$ NMR [39-42,48] and of MD simulations [50,51]. From the ${ }^{2} \mathrm{H}$ NMR investigation, the THF dynamics occur in the rapid regime (i.e. with characteristic time shorter than the nanosecond) at temperatures above ca. $100 \mathrm{~K}$. Moreover, the NMR results suggest that the THF reorientations within the cage are not isotropic: it may exist an anisotropic character of the THF dynamics induced by a distribution of the local environment. Neutron powder diffraction study reveals the existence of preferred orientations of the THF within the cages [32], thus confirming the NMR conclusions. Finally, MD simulations show the existence of H-bond between the oxygen atoms of THF molecules and the hydrogen atoms of the water molecule with a lifetime of less than 1 ps [50]. Such hydrogen bonding is particularly relevant in the understanding of the mechanism of clathrate formation [52].

The aim of this paper is to investigate the THF dynamics encapsulated within the cages of its type II clathrate, by combining quasielastic neutron scattering (QENS) and molecular dynamics (MD) investigations. Such combined methodology is particularly appropriated for investigating guest dynamics in clathrate hydrates [53-54]. This investigation provides new insight onto the THF dynamics with complementary timescale with respect to previous investigations by means of NMR [37-42,47-49], such information is essential to understand guest-water interactions in view of the extended timescale met in the clathrate hydrates [54]. Moreover, MD-QENS combined analyzis consists in a "direct showdown" of experimental and theoretical results through the comparison of QENS-measured and MD-derived scattering laws, leading to the validation of the potential used in the MD simulations. Molecular dynamics simulations of the THF clathrate hydrate are then used to guide the interpretation of the experimental QENS spectra. Thus, such a combined approach has been used for the first time in this system and yields unique information about the THF molecule dynamics and its orientational distribution within the cages.

\section{Neutron scattering and molecular dynamics details}

\subsection{QENS experiments}

The samples have been prepared by mixing deuterated water (99.5\% deuterated) and tetrahydrofuran (noted THF) in stoichiometric proportion (17:1 mol). The prepared solution has been stirred in a thermal bath at a constant temperature of $274 \mathrm{~K}$ (the melting point of the THF clathrate hydrate is $277 \mathrm{~K}$ ) until crystallization occurs (in about one day). Under cold nitrogen atmosphere, the resulting THF clathrate hydrate has been grounded to form a fine powder and filled into flat aluminium containers sealed with indium wire to perform the QENS experiments.

QENS experiments with the THF- $17 \mathrm{D}_{2} \mathrm{O}$ sample have been performed using the time-of-flight (denoted ToF) spectrometer IN5 [55] at the Institut Laue Langevin (Grenoble, France). The scattering angles covered by this instrument are in the range $14^{\circ}-132^{\circ}$ and the energy resolution was $\Delta E \sim 90 \mu \mathrm{eV}$ for an incident wavelength $\lambda_{\mathrm{o}}=5 \AA$. The samples have been cold-transferred into the cryostat of the spectrometer and the angle between the incoming neutron beam and the plane of the sample holder was $135^{\circ}$. Minimization of the multiple scattering effects is reached by using a sample thickness less than $0.2 \mathrm{~mm}$, so that the effective transmitted beam corresponds to about $97 \%$ of the incident beam. QENS spectra of the THF clathrate hydrate have been recorded at $50 \mathrm{~K}, 100 \mathrm{~K}$, $150 \mathrm{~K}, 200 \mathrm{~K}$ and $270 \mathrm{~K}$ with acquisition time of $1 \mathrm{~h}$ per temperature. Background corrections have been performed by recording empty cell spectra and a vanadium spectrum has been used to correct for detector efficiency and to determine the experimental res- olution function. Two sets of data have been extracted from the neutron scattering experiments. For the structural analyzis, only elastic scattering has been extracted from the experimental data at each scattering angle. For QENS analyzis, several detectors were grouped together to improve the statistical accuracy and Bragg peaks were removed from experimental data. The QENS data analyzis has been done by using the NEMO program [56].

The sample integrity has been checked with the help of the diffraction patterns of the THF clathrate hydrate recorded with IN5 (Fig. 1). In the studied temperature range, no structural phase transition has been observed and all Bragg peaks have been indexed with the unit cell characteristic of the type II clathrate structure (i.e. cubic unit cell with $\mathrm{a} \approx 17.2$ A and space group $F d 3 m$ ).

\section{2. $M D$ details}

To reproduce the interaction between all particles in the systems, the rigid and pairwise additive potential function used in these simulations is constituted of a Lennard-Jones type potential combined with an electrostatic point charge potential. The interaction energy between two molecules in the system is thus expressed as a sum of interactions between atoms $\alpha$ of molecule $A$ and atoms $\beta$ of molecule $B$ separated by the distance $r_{\alpha \beta}$,

$U\left(r_{\alpha}, r_{\beta}\right)=\sum_{\alpha \in A} \sum_{\beta \in B}\left\{4 \varepsilon_{\alpha \beta}\left[\left(\frac{\sigma_{\alpha \beta}}{r_{\alpha \beta}}\right)^{12}-\left(\frac{\sigma_{\alpha \beta}}{r_{\alpha \beta}}\right)^{6}\right]+\frac{q_{\alpha} q_{\beta}}{r_{\alpha \beta}}\right\}$,

In this equation, $\varepsilon_{\alpha \beta}$ and $\sigma_{\alpha \beta}$ are the Lennard-Jones parameters computed according to the geometric mixing rules and $q_{\alpha}$ is the partial charge located on atom $\alpha$. The SPC/E model of Berendsen et al. is used to describe water [57], as in previous MD simulations of THF clathrate hydrate [50]. For the THF molecule, a rigid five sites model has been used by considering $\mathrm{CH}_{2}$ groups as single sites. The structure of the THF molecule has been determined by electronic structure calculations with the Gaussian 98 program package [58]. In order to compare with a previous work [50], geometry optimization have been performed using the DFT method with the B3LYP functional and the $6-311++G(d, p)$ basis set [59]. The atomic charges have been fitted to reproduce the electrostatic potential of the molecule obtained from ab initio at the same level of calculation by means of the CHELPG method [60]. To obtain the charges of single $\mathrm{CH}_{2}$ groups, the hydrogen charges

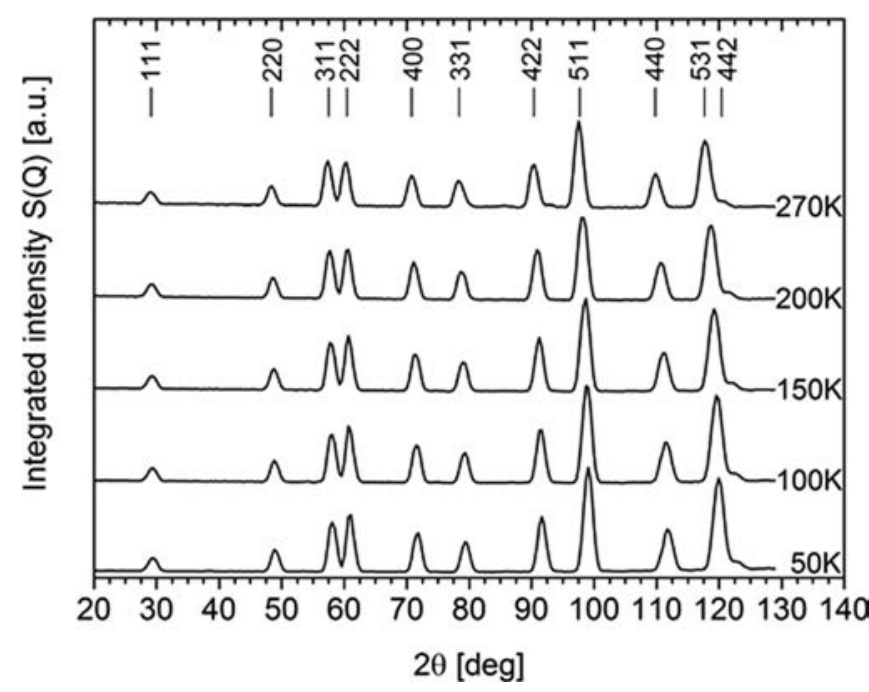

Fig. 1. Powder diffractograms of the THF clathrates hydrates recorded at indicated temperatures with the ToF spectrometer IN5@ILL $\left(\lambda_{0}=5 \AA\right)$. The Bragg peaks are indexed in the type II clathrate structure (i.e. space group Fd3 $m$ with a $\approx 17.2 \AA$ ). 
Table 1

Values of the parameters used in the intermolecular interaction potential (see text for details). Original SPC/E parameters for water [57] and ab initio derived parameters for THF molecule.

\begin{tabular}{llll}
\hline Atom & $\varepsilon[\mathrm{kJ} / \mathrm{mol}]$ & $\sigma[\AA]$ & $q[\mathrm{e}]$ \\
\hline Water molecule & & & \\
O & 0.65017 & 3.16556 & -0.8476 \\
$\mathrm{H}$ & 0 & 0 & 0.4238 \\
THF molecule & & & \\
C1 & 0.4577 & 3.400 & +0.2226 \\
O2 & 0.7113 & 3.000 & -0.4660 \\
C3 & 0.4577 & 3.400 & +0.0120 \\
C4 & 0.4577 & 3.400 & +0.2214 \\
C5 & 0.4577 & 3.400 & +0.0100 \\
\hline
\end{tabular}

have been added to the corresponding carbon atom. The LennardJones parameters have been taken from the general AMBER force field [61]. The values of these parameters are given in the Table 1 and the numbering of carbon atoms is shown in Fig. 2.

The initial type II clathrate structure has been generated by using the fractional coordinates determined by means of X-ray diffraction experiments [31]. The primary cell has been duplicated in the three directions of the space to give a cubic simulation box with an edge of $34 \AA$ and containing 1088 deuterated water molecules and 64 THF molecules (located in large cages). The center of mass of the THF molecules was originally placed at the center of mass of the large cages with random orientation. Usual periodic boundary conditions have been used and a molecular cut off distance of 16 Å has been applied. No method has been used to handle the long-range electrostatic interactions. The simulations were carried out using the MDpol package [62], particularly appropriated for combined MD-QENS analyzis of organic nanoporous systems [63-65]. The calculations have been performed in the NPT ensemble by using the Nosé-Andersen algorithm (with coupling constants of $4.0 \mathrm{ps}^{-1}$ and $0.9 \mathrm{ps}^{-1}$ for the heat bath and the piston,

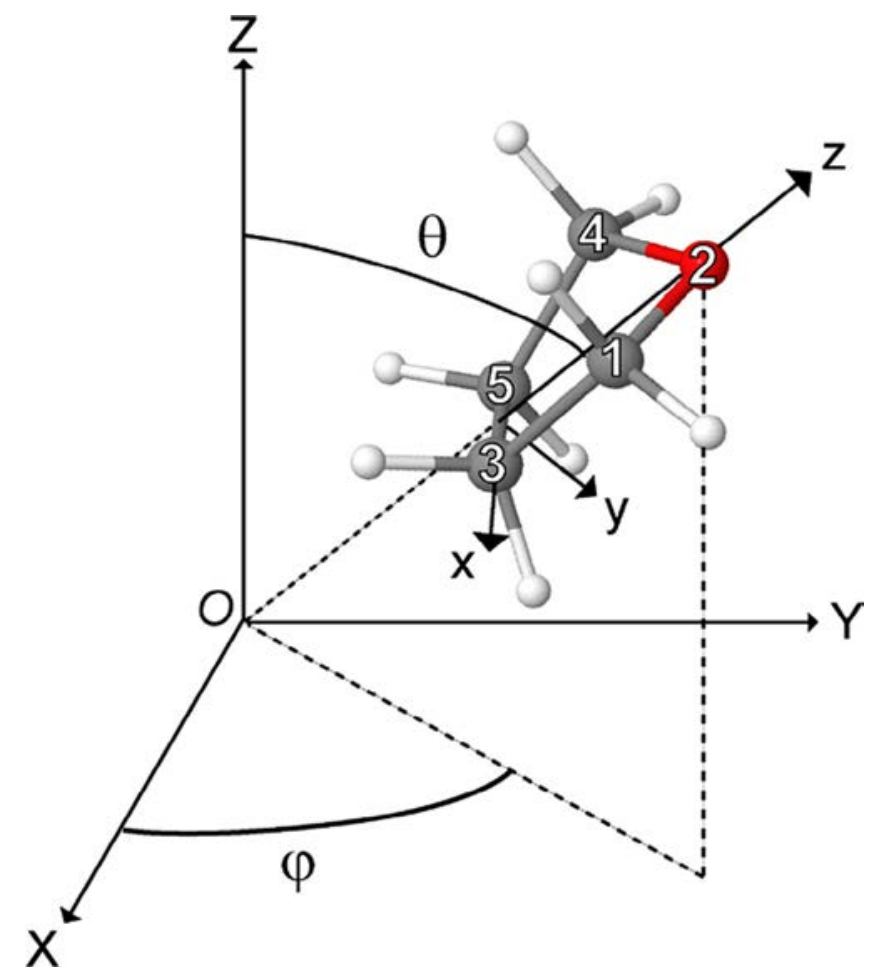

Fig. 2. Representation of THF molecular frame (Cxyz) as well as the polar $(\theta)$ and azimuth $(\varphi)$ angles with respect of the MD box reference frame (OXYZ).

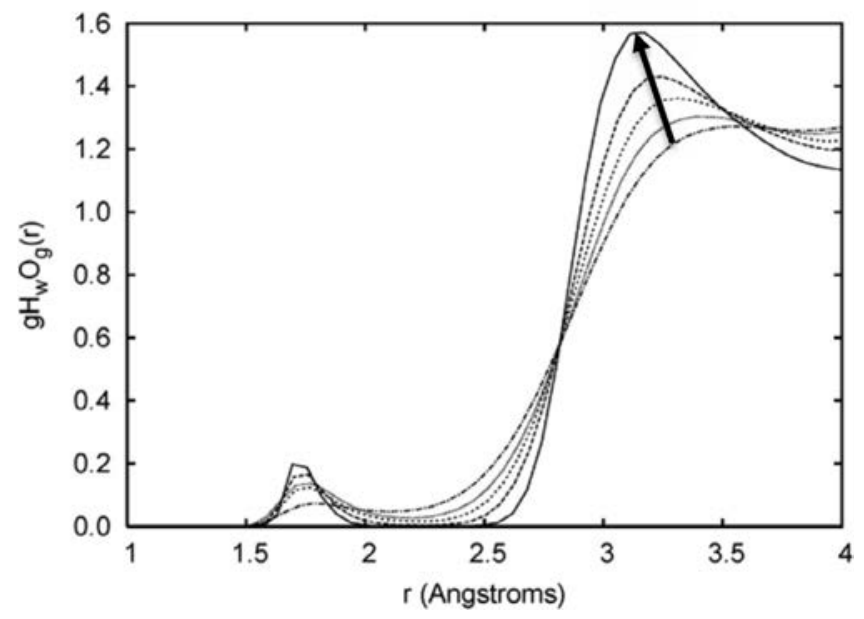

Fig. 3. Pair distribution function of the oxygen atom on the THF molecules with the lattice water hydrogen atoms at $50 \mathrm{~K}, 100 \mathrm{~K}, 150 \mathrm{~K}, 200 \mathrm{~K}$ and $270 \mathrm{~K}$ (the arrow indicates decreasing temperatures).

respectively) $[66,67]$ modified to take into account the necessary constraints that maintain rigid the molecules. The equations of motion were integrated with a time step of $1 \mathrm{fs}$ using the Verlet leapfrog scheme [68]. Each system has been equilibrated for a time of 500 ps used for temperature and pressure scaled equilibration. In order to be consistent with the QENS investigations, trajectories have been produced during runs of $500 \mathrm{ps}$ at temperatures between $50 \mathrm{~K}$ and $270 \mathrm{~K}$ and at a pressure of 1 bar.

The integrity of the simulated system has been checked through the calculations of various radial pair distribution functions, confirming the inclusion of THF molecules exhibiting no long range translational diffusion and located within the large cages formed by means of a tetrahedral H-bond network of water molecules (see supplementary material). Among the radial pair distribution functions, the one calculated according to the distance between the oxygen atom of the THF molecule and the water hydrogen atoms (Fig. 3) exhibits a small peak at ca. $1.75 \AA$. As analyzed in previous MD analyzis [50,51], such a peak is a direct signature of the known formation of host-guest hydrogen bonding.

\section{Results and discussion}

By performing a neutron scattering experiment, the measurement of the probability to find a neutron leaving the sample is done in a given direction within a solid angle element and with a given energy [69]. This double differential scattering cross section can be reduced to an experimental scattering law, $S_{\text {exp }}(\boldsymbol{Q} \omega)$, recorded as a function of momentum transfer $\hbar \mathbf{Q}$ and of the energy transfer $\hbar \omega$. This law consists of the superimposition (weighted by the incoherent and coherent scattering cross sections of the scatterers) of the incoherent scattering (related to auto-correlation) providing information about the motion of individual atoms and of the coherent scattering (related to crossed correlation) providing information about concerted or correlated motions. The present sample has been prepared by deuterating the host water substructure, the THF being hydrogenated. The incoherent cross section of deuterium is 40 times less than the one of hydrogen. The incoherent scattering of the water cages is then minimized and the coherent contributions can be neglected, since Bragg peaks were removed from the experimental data and coherent inelastic scattering is well separated from the quasielastic region of the spectra. It follows that the present QENS experiments give access to the incoherent signal arising from the THF molecules and thus to the THF dynamics. 
In order to develop a comprehensive model of the THF dynamics, we will combine the MD and QENS results. In this purpose, the MD simulations results will be first compared to the QENS results by computing the MD-derived incoherent neutron scattering laws with the help of the atomic MD trajectories. Once this validation step will be done, a model reproducing the THF dynamics will be developed with the help of the MD trajectories.

\subsection{Comparison of the experimental and $M D$-derived neutron scattering laws}

In the quasi-elastic region (i.e. for energy transfer of $\pm 3 \mathrm{meV}$ ), the relationship between the measured QENS spectra, $S_{\text {exp }}(\boldsymbol{Q} \omega)$, and the clathrate hydrate scattering law, $S(\boldsymbol{Q} \omega)$, is given by $[69,70]$,

$S_{\text {exp }}(\boldsymbol{Q}, \omega)=F(Q) e^{-\hbar \omega / k_{B} T} S(\boldsymbol{Q}, \omega) \otimes R(\boldsymbol{Q}, \omega)+B(\boldsymbol{Q})$,

where $F(\boldsymbol{Q})$ is the scaling factor depending on the momentum transfer $Q$ through the Debye-Waller factor $\left(e^{-} Q^{2}\left\langle u^{2}\right\rangle\right.$ where $\left\langle u^{2}\right\rangle$ is the mean square displacement), $T$ is the temperature and $k_{B}$ is the Boltzmann constant. $B(\boldsymbol{Q})$ is the background term reproducing the inelastic contributions in the quasi-elastic region. The function $R$ $(\boldsymbol{Q} \omega)$ represents the experimental energy resolution. The width of the energy resolution function $(\Delta \mathrm{E}=90 \mu \mathrm{eV}$ in the present experiment) gives the observation timescale (of the order of picosecond in the present case) on which a given motion will be observed on the experimental spectra. Any dynamical process, occurring on a timescale significantly longer than the observation time, will give rise to a QENS broadening narrower than the instrumental resolution function and the experimental scattering law reduces to a purely elastic component. The incoherent scattering law due to the clathrate hydrate, $S(\boldsymbol{Q} \omega)$, is the normalized sum of the incoherent contribution of the THF molecule and of the incoherent contribution of the cage water molecule,

$S(\boldsymbol{Q}, \omega)=(1-p) S_{T H F}(\boldsymbol{Q}, \omega)+p S_{\text {water }}(\mathbf{Q}, \omega)$.

One needs to determine the contribution of deuterated cage substructure in the QENS spectra since the deuterium nuclei possesses an incoherent cross section. The reorientation timescale of the water molecules of the aqueous sub-structure is of the order of microsecond [39-41], i.e. significantly longer than the observation time. Thus, the water molecules will be immobile with respect to the probed timescale, and the host sub-structure will reduce to an elastic contribution to the scattering law,

$S(\boldsymbol{Q}, \omega)=(1-p) S_{T H F}(\boldsymbol{Q}, \omega)+p \delta(\omega)$,

where $S_{T H F}(\mathbf{Q} \omega)$ is the incoherent neutron scattering law due to the guest molecules, $\delta(\omega)$ is a Dirac function due to elastic scattering of the water molecules and $p$ is the corresponding incoherent scattering fraction. Taking into account for the $99.5 \%$ deuteration and for the guest to host molecular ratio of $1: 17$, the incoherent contribution of the deuterated water molecules is contained in the elastic term $p$, representing $11.5 \%$ of the incoherent signal of the THF clathrate hydrate sample (i.e. $p=0.115$ in Eq. (4)).

Computing the powder averaged intermediate scattering function derived from the MD trajectories (the program nMoldyn has been used [71]) makes accessible the comparison of the time-offlight QENS data with the MD-simulations data. Following the van Hove formalism [69], the MD-derived intermediate scattering law has been Fourier transformed according to the following expression,

$$
\begin{aligned}
S_{M D}(\boldsymbol{Q}, \omega) & =F(\boldsymbol{Q}) \frac{1}{2 \pi} \int_{-\infty}^{+\infty} R(\boldsymbol{Q}, t)\left[(1-p) I_{T H F}^{M D}(\boldsymbol{Q}, t)+p\right] e^{-i \omega t} d t \\
& =F(\mathbf{Q})\left[(1-p) S_{T H F}^{M D}(\boldsymbol{Q}, \omega)+p \delta(\omega)\right] .
\end{aligned}
$$

In this expression, the MD-derived intermediate scattering function $I_{T H F}^{M D}(\boldsymbol{Q}, t)$ and MD-derived scattering law $S_{T H F}^{M D}(\boldsymbol{Q}, \omega)$ represent the incoherent contribution of the THF molecules (in the time domain and in the energy domain, respectively). As previously mentioned, the water molecules undergo reorientations on a timescale longer than the observation time reached by means of QENS experiments and also by means of MD simulations (see water orientational autocorrelation functions provided in the supplementary material), so that no quasielastic broadening due to the water molecules dynamics is expected on the probed MD timescale. The water molecule contribution is then taken into account through the Dirac function weighted with the incoherent fraction $p$. Finally, the MD-derived intermediate scattering function is folded with a Gaussian function (in the time domain) determined with the help of the Fourier transform of the experimental vanadium QENS spectra for reproducing the experimental conditions.

In both set of data, i.e. the experimental and MD-derived QENS spectra, the incoherent contribution arising from the guest molecules have been fitted with the help of the following phenomenological scattering law,

$S_{T H F}(\boldsymbol{Q}, \omega)=A_{0}(\mathbf{Q}) \delta(\omega)+\left[1-A_{0}(\mathbf{Q})\right] \frac{1}{\pi} \frac{\Delta}{\Delta^{2}+\hbar \omega}$,

where $A_{0}(\mathbf{Q})$ is the elastic incoherent structure factor (EISF) giving the amplitude of the elastic term represented by a Dirac function $\delta(\omega)$ and provides information about the geometry of the THF dynamics. Both MD-derived and experimental neutron scattering laws have been fitted with the expression (6) as shown in Fig. 4. The Lorentzian function represents the averaged quasielastic contributions for which the half-width at half-maximum (denoted HWHM), $\Delta$, provide information about the characteristic time of the THF motion. The measured HWHMs (Fig. 5) have been averaged over the whole $\boldsymbol{Q}$ range for allowing direct comparison of the MDderived and experimental broadenings. Two temperature ranges are discriminated in both set of data with the inflection point observed at $c a .100 \mathrm{~K}$. In the high temperature region (i.e. above ca. $100 \mathrm{~K}$ ), there is a good agreement between both set of data. The associated relaxation process is characterized with identical thermal behavior of the HWHMs (with an overall small offset of the MD-derived HWHM with respect to the experimental HWHMs) and with identical structure factors (see Fig. 6) in the theoretical and experimental data. In the low temperature region (i.e. below ca. $100 \mathrm{~K}$ ), the relaxation process is not activated at the same temperatures in both sets of data (the slope of the MD-derived and experimental QENS HWHMs are different). These behaviors result in slight differences between the experimental EISFs and the MDderived EISFs in the intermediate temperature region at $c a .100 \mathrm{~K}$ (Fig. 6). Nevertheless, the relaxation process is characterized by means of experimental structure factor well reproduced by the MD-derived structure factor (see Fig. 6) at $50 \mathrm{~K}$. Both MD-derived and QENS EISFs are closed to 1, suggesting that the THF QENS broadening is not correctly resolved with respect to the present energy resolution. At $50 \mathrm{~K}$, this behavior indicates that the THF dynamics occur on a timescale significantly longer than the probed timescale, i.e. with a characteristic time longer than the picosecond. Such a timescale is in full agreement with dielectric spectroscopy [34], ${ }^{1} \mathrm{H}$ NMR [37,47-49] and ${ }^{2} \mathrm{H}$ NMR [39-42,48], according to these measurements the characteristic time for THF reorientation is shorter than $10^{-10} \mathrm{~s}$ for temperature above $c a .100 \mathrm{~K}$.

Thus, the MD trajectories lead to spatial and time characteristics of the THF dynamics similar to those observed with the help of QENS experiments. As observed with the analyzis of the structure factors, no long-range diffusion of the THF molecules is observed on the probed timescale. Only reorientations are observed on a timescale of the order of the picosecond above $c a$. 
MD-derived QENS spectra
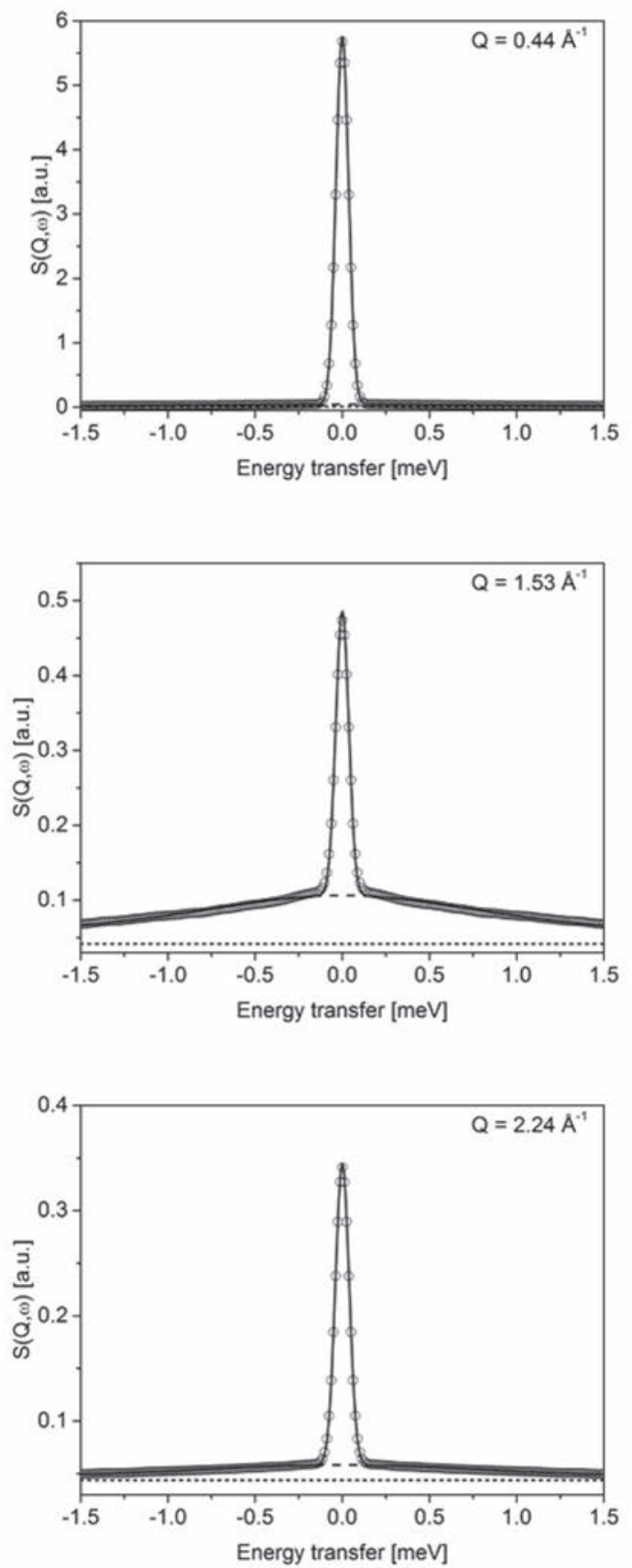

Experimental QENS spectra
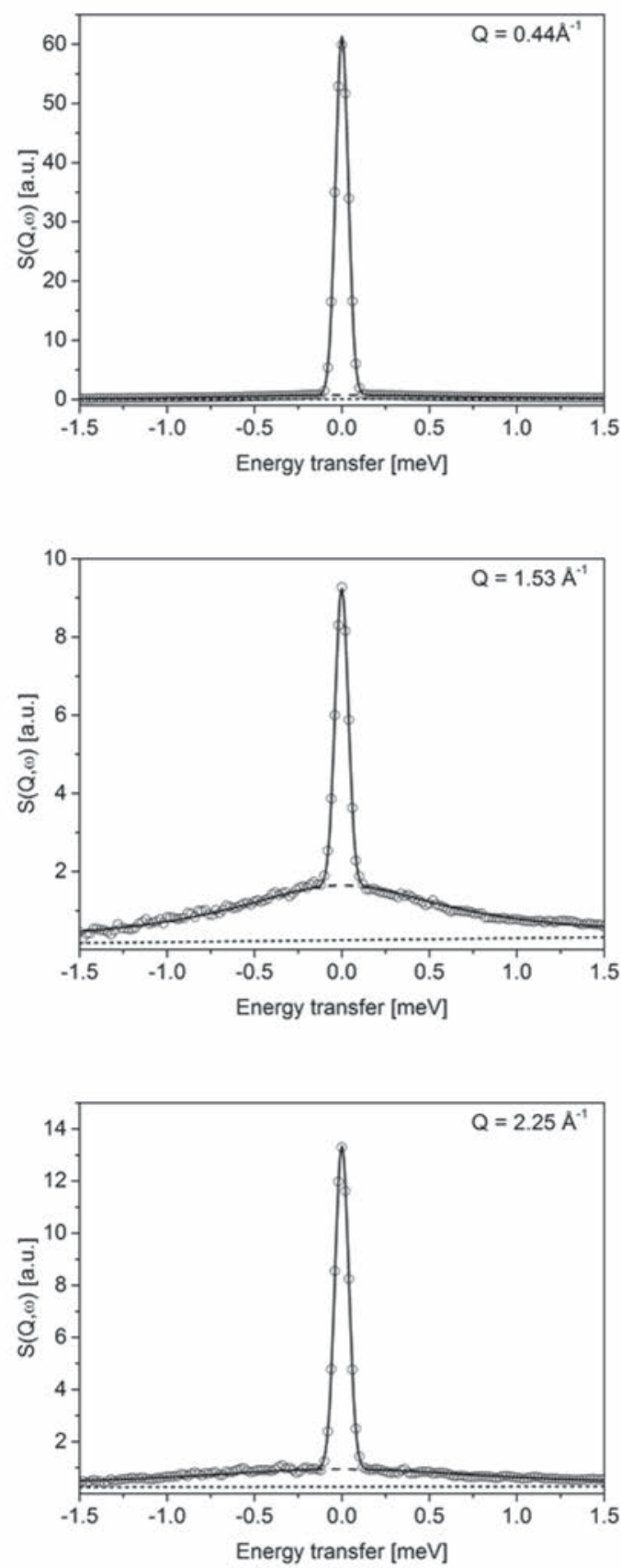

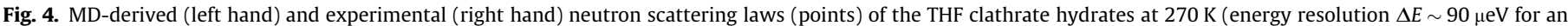

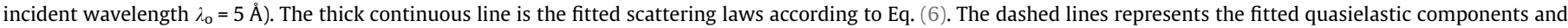
the dotted lines, scattering backgrounds.

$100 \mathrm{~K}$ on both MD-derived and QENS scattering laws. Such an agreement between the experimental and theoretical data allows the validation of the MD simulations and thus, the subsequent analyzis of the MD trajectories in order to elaborate a detailed model of the THF dynamical process.

\subsection{Orientational distribution functions of the THF molecules}

The calculation of the orientational distribution functions (denoted ODFs in the following) requires the definition of a molecular frame (Cxyz) with respect to the MD box reference frame
(OXYZ) as represented in Fig. 2: the (CX) axis is co-linear to the $\mathrm{C} 5-\mathrm{C} 3$ bond and the $(\mathrm{Cz})$ axis goes through the oxygen atom and the middle of the $\mathrm{C} 3-\mathrm{C} 5$ bond (denoted $\mathrm{C}$, the origin of the molecular frame). The latter axis corresponds to the $C_{2}$ symmetry axis. In addition, three specific angles have been used by following the Euler definition. The polar $\theta$ and azimuth $\varphi$ angles of the $(C z)$ molecular axis will be used to describe the orientation of the THF molecules within the $5^{12} 6^{4}$ cages. In addition, the third Euler angle $\psi$ (not represented in Fig. 2) has been defined to explore the rotation of the THF molecule about the $(\mathrm{Cz})$ molecular axis. This angle has been computed with the help of the following procedure. At 


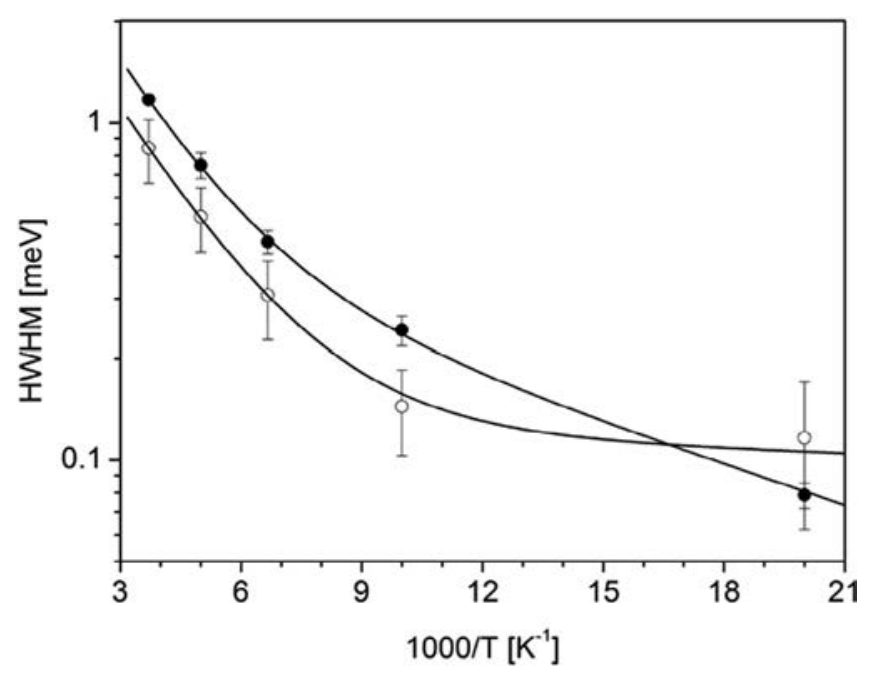

Fig. 5. Experimental (open symbols) and MD-derived (filled symbols) HWHM of the THF clathrate hydrates at various temperatures (energy resolution $\Delta E \sim 90 \mu \mathrm{eV}$ for an incident wavelength $\lambda_{\mathrm{o}}=5 \AA$ ) averaged over momentum transfers. The continuous lines represent guide to the eyes.

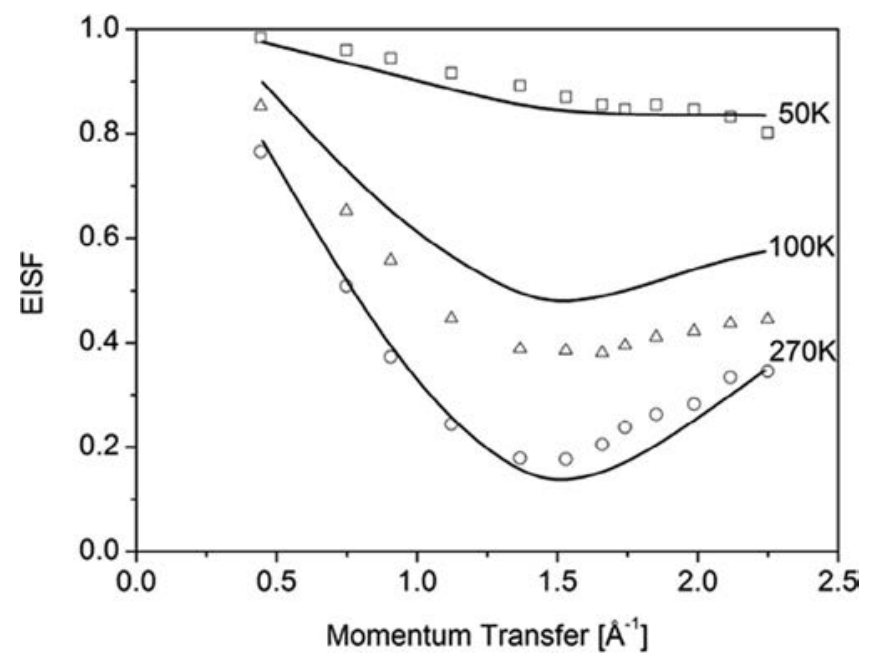

Fig. 6. Experimental (points) and MD-derived (lines) EISF of the THF clathrate hydrates at various temperatures (energy resolution $\Delta E \sim 90 \mu \mathrm{eV}$ for an incident wavelength $\lambda_{\mathrm{o}}=5 \AA$ ).

each time-step, the azimuth and polar angles have been calculated and the THF molecules have been rotated by the angle $\varphi$ about the $(\mathrm{OZ})$ axis and then by the angle $\theta$ about the (OY) axis. The angle between the (OX) axis and the C3-C5 bond (i.e. $\psi$ ) has then been calculated.

By considering the $N_{\text {step }}$ time-steps and the $N_{\text {mol }}$ THF molecules of the MD simulations, the normalized orientational distribution function (denoted ODF in the following) $P(\psi)$ has been calculated by using the expression,

$P(\psi)=\frac{1}{N_{\text {step }} N_{\text {mol }}} \sum_{1}^{N_{\text {step }} N_{\text {mol }}} \sum_{1} N(\psi)$ with $\int P(\psi) d \psi=1$,

where $N(\psi)$ is the number of THF molecules with angle $\psi$ in the range $[\psi, \psi+d \psi]$. The resulting ODF $P(\psi)$ calculated by using the MD trajectories at $270 \mathrm{~K}$ is shown in Fig. 7. This ODF is constant as a function of $\psi$. This indicates that the THF molecules undergo continuous rotations about the $(\mathrm{Cz})$ molecular axis at $\mathrm{T}=270 \mathrm{~K}$. By decreasing the temperature to $50 \mathrm{~K}$, the ODF $P(\psi)$ exhibits a

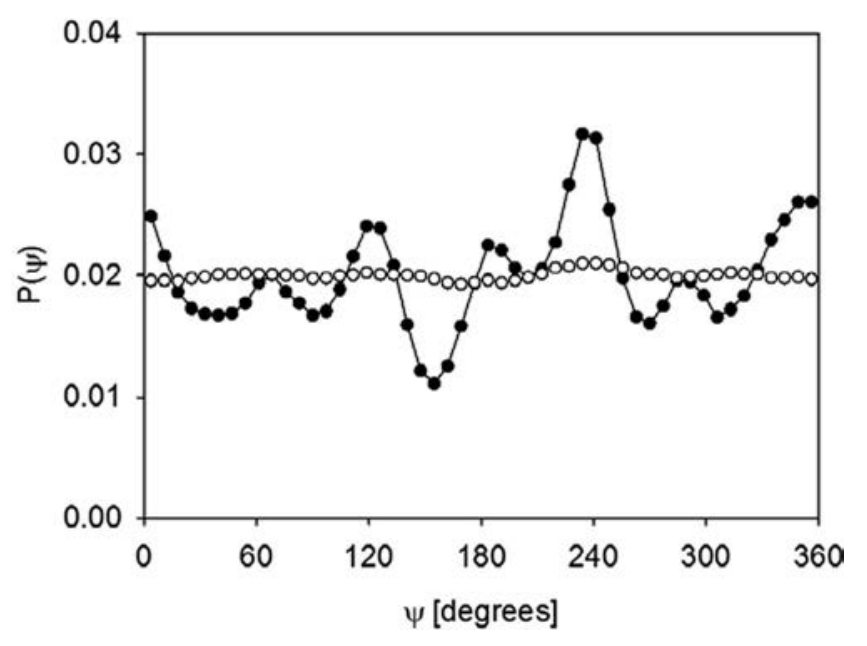

Fig. 7. Normalized orientational distribution function $P(\psi)$ of THF molecule located in large $5^{12} 6^{4}$ cages of the type II clathrate structure at $T=270 \mathrm{~K}$ (open symbols) and at $\mathrm{T}=50 \mathrm{~K}$ (filled symbols).

more structured shape: there are six maxima unequally weighted. Such ODF can be representative of a static or dynamic disorder: either the THF molecule is stuck in one $\psi$ orientation with a static distribution of these orientations differing from cage to cage or the THF molecule undergoes rotation about its $(\mathrm{Cz})$ axis between 6 unequiprobable orientations. According to the phenomenological analyzis, the THF dynamics below $100 \mathrm{~K}$ occur on a timescale accessible by means of NMR, i.e. longer than the one probed in the present MD-QENS analyzis. With respect to the probed timescale, the static distribution of the preferred orientations is the most plausible.

In order to analyze whole reorientations of the THF molecules, the ODF $P(\theta, \varphi)$ of the $(\mathrm{Cz})$ axis have also been calculated by using

$P(\theta, \psi)=\frac{1}{N_{\text {step }} N_{\text {mol }}} \sum_{1}^{N_{\text {step }} N_{\text {mol }}} \sum_{1} \frac{N(\theta, \psi)}{\sin \theta}$ with $\iint P(\theta, \psi) \sin \theta d \theta d \psi=1$,

where $N(\theta, \varphi)$ is the number of THF molecules with azimuth angle $\varphi$ in the range $[\varphi, \varphi+d \varphi]$ and polar angle $\theta$ with $\cos (\theta)$ in the range $[\cos (\theta), \cos (\theta)+d \cos (\theta)]$. The calculated ODFs are shown in Fig. 8 for all THF molecules of the MD simulations box at $\mathrm{T}=270 \mathrm{~K}$ and at $\mathrm{T}=50 \mathrm{~K}$. None of these ODFs respects the $T_{d}$ symmetry of the $5^{12} 6^{4}$ cage occupied by the THF molecule. Indeed, in such a case, one would have expected to observe four equally weighted maxima on these distributions. Instead, one preferred orientation (at least) of the $(C z)$ axis is observed $\left(\theta \sim 90^{\circ}\right.$ and $\varphi \sim 180^{\circ}$ at $\left.\mathrm{T}=270 \mathrm{~K}\right)$ as pointing toward the hydrogen bonds of the pentagonal faces. Such ODFs are in agreement with previous results: deuteron NMR analyzis [42] has revealed the existence of a "distorted octahedral" reorientational model, i.e. not respecting the $T_{d}$ symmetry of the cage encapsulating the THF molecules and Neutron Powder Diffraction [32] analyzis has showed the existence of preferred orientation adopted by the THF molecule. Moreover, analyzis of the ODF of individual THF molecule shows that there is a distribution of these preferred orientations. These various local environments lead to ODF at $\mathrm{T}=270 \mathrm{~K}$ for which all orientations are adopted: the ODF is quite smooth with probabilities ranging between $8.010^{-6}$ and $1.810^{-5}$. Thus, the THF molecule undergoes quasi-isotropic reorientations with some anisotropic character at $\mathrm{T}=270 \mathrm{~K}$. At $\mathrm{T}=50 \mathrm{~K}$, the preferred orientations are more pronounced and all orientations are not experienced by the THF molecules. The dynamical exchange between the preferred orientations might be frozen with respect 


\section{$\mathrm{T}=270 \mathrm{~K}$}

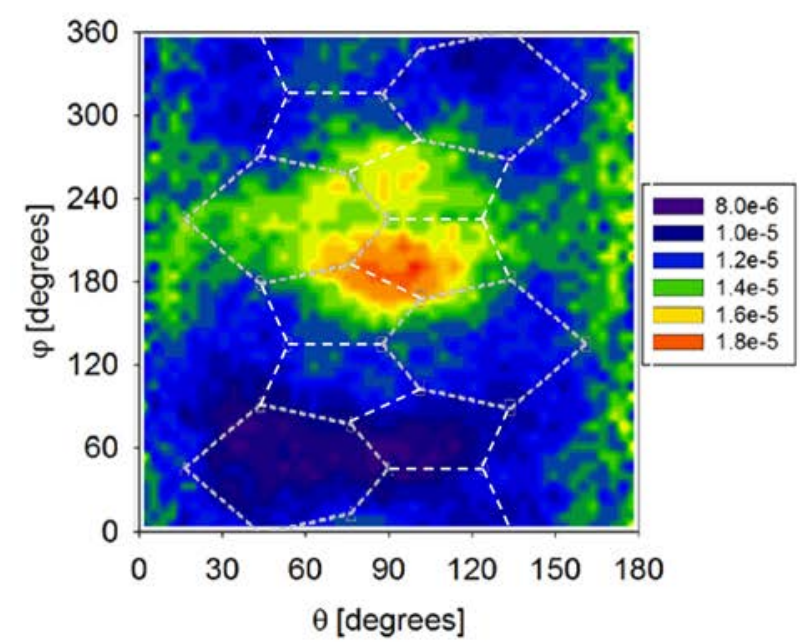

\section{$\mathrm{T}=50 \mathrm{~K}$}

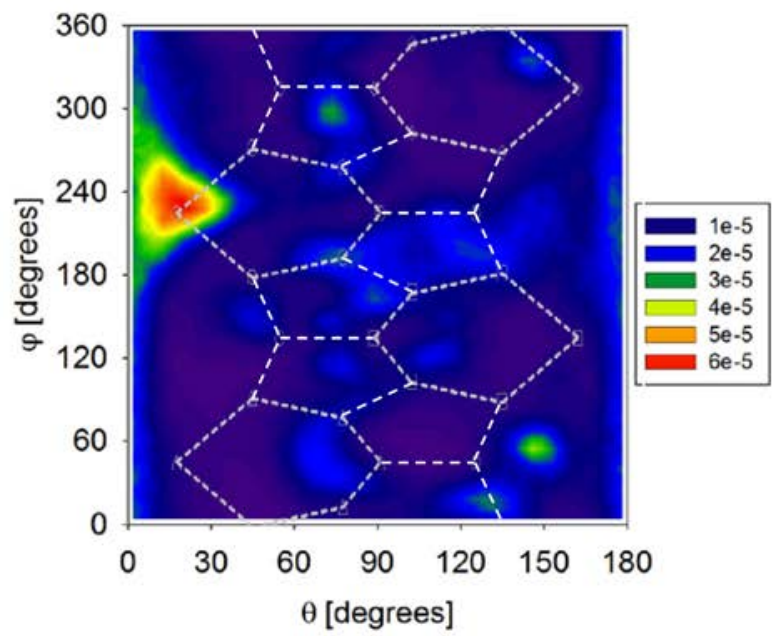

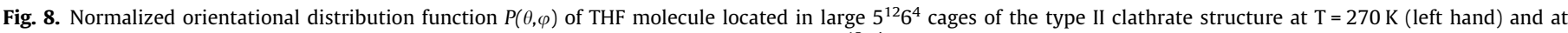
$\mathrm{T}=50 \mathrm{~K}$ (right hand). The dashed lines represent a projection of the water H-bonds of the $5^{12} 6^{4}$ cage (the oxygen atoms are at the corners of the polygons).

of the MD observation time according to the phenomenological analyzis done in the previous section.

As a summary of the analyzis of the ODFs and of the phenomenological analyzis of QENS and MD-derived scattering laws, the main characteristics of the THF dynamics may be described into two types of motions:

- the THF molecule undergoes rotation about its $C_{2}(\mathrm{Cz})$ molecular axis (Fig. 2). By lowering the temperature from $270 \mathrm{~K}$ to $50 \mathrm{~K}$, this motion evolves from a continuous rotation to modulated rotation occurring on a timescale longer than the one probed in the present MD-QENS investigation at $50 \mathrm{~K}$.

- The $C_{2}$ molecular axis performs slightly anisotropic reorientations with preferred orientations in the cage, pointing toward the hydrogen bonds of the pentagonal faces. By lowering the temperature to $50 \mathrm{~K}$, the THF molecules get frozen in preferred orientation with respect to the probed timescale.

\subsection{Orientational relaxation processes of the THF molecules}

The subsequent step in analyzing the THF dynamics concerns the determination of the timescale of the various dynamical processes. In this issue, the autocorrelation functions of the three THF molecular axis $\langle x(0) \cdot x(t)\rangle,\langle y(0) \cdot y(t)\rangle$ and $\langle z(0) \cdot z(t)\rangle$ have been computed and are represented on the Fig. 9. In the frame of the description done on the basis of the ODFs, the $\langle z(0) . z(t)\rangle$ autocorrelation function is representative of the relaxation within the $P(\theta$, $\varphi)$ ODF (motion (ii) previously described as the quasi-isotropic reorientation) while the $\langle x(0) \cdot x(t)\rangle$ and $\langle y(0) \cdot y(t)\rangle$ autocorrelation functions allow the relaxation within the $P(\psi)$ ODF (motion (i) previously described as the rotations about the $(\mathrm{Cz})$ axis) to be probed. The features of these three functions (Fig. 9) confirms this description: the $\langle z(0) \cdot z(t)\rangle$ time dependence differs from $\langle x(0) \cdot x(t)>$ and $<y$ $(0) \cdot y(t)>$ time dependences. The $\langle z(0) . z(t)>$ function does not relax to zero at long time (compared to the MD timescale), suggesting no
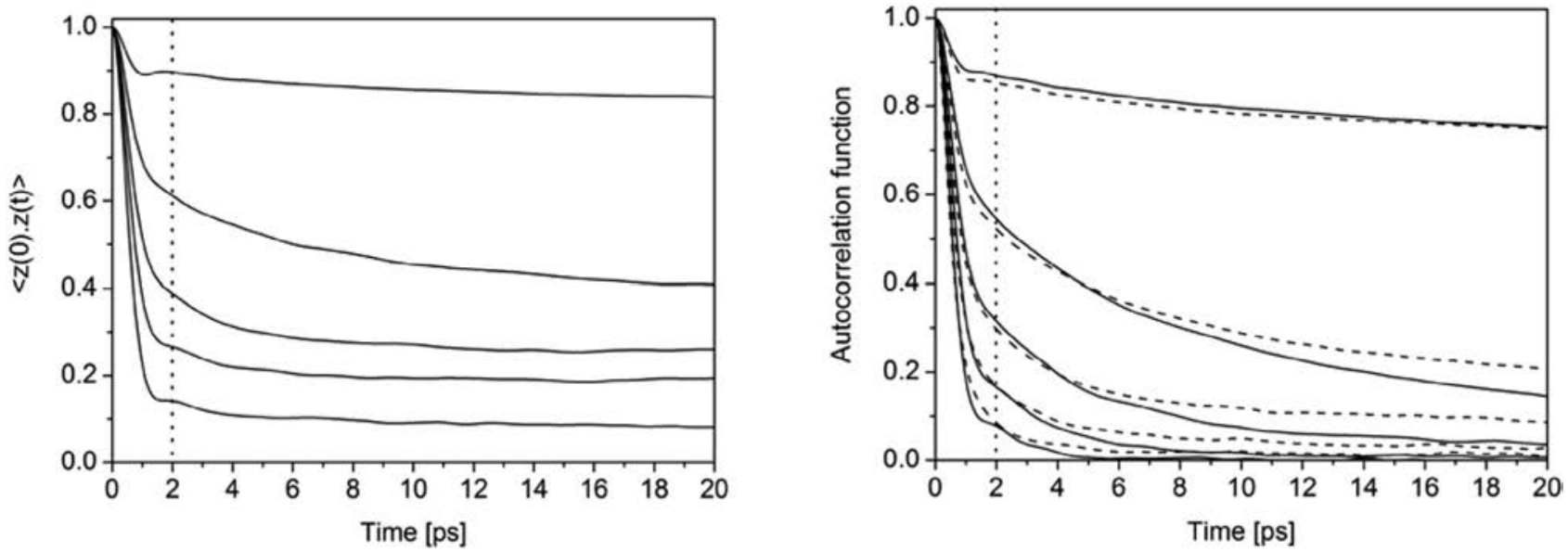

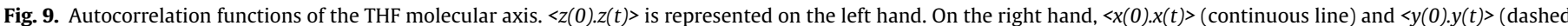

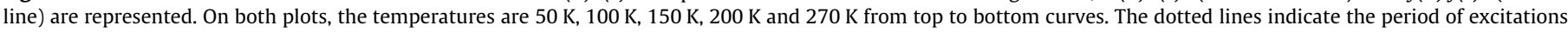
observed on autocorrelation functions (see text for details). 
full loss of correlation of the $(C z)$ molecular axis. Such behavior is in agreement with the existence of preferred orientation as observed on the $P(\theta, \varphi)$ ODF. Both $\langle x(0) \cdot x(t)>$ and $\langle y(0) \cdot y(t)>$ functions follow the same behavior. Slight differences are observed at long time. However, the loss of correlation is reached within the probed time range for the two highest temperatures. Focusing on the temperature dependence, it appears that the THF molecule relax on a significant long timescale at $50 \mathrm{~K}$. This behavior confirms the fact that, at $50 \mathrm{~K}$, the THF molecules are frozen (with respect to MD and QENS timescales) in preferred orientations statically distributed. Finally, these autocorrelation functions are clearly not decaying as a single exponential. One can observe the existence of a rotational excitation (oscillating signal observed at short time) in addition to the relaxation phenomenon, as observed in other type II clathrate hydrate [53]. This excitation is highly damped: a single oscillation is observed with a time period of ca. 2 ps (indicated by a vertical dotted line on Fig. 9). Moreover, this excitation is less pronounced on the $\langle y(0) \cdot y(t)\rangle$ autocorrelation function. This observation suggests that this excitation is characteristic of a dynamical process of the THF molecule about the (Cy) axis, i.e. an in-plane rotational excitation.

A quantitative analyzis of the autocorrelation functions has been performed without explicitly considering the in-plane excitation. Considering a single decaying exponential function could not satisfactorily reproduce the autocorrelation functions. It was necessary to consider two exponential functions,

$C(t)=A_{1} \exp \left(-\frac{t}{\tau_{1}}\right)+A_{2} \exp \left(-\frac{t}{\tau_{2}}\right)+A_{3}$ with $\sum_{i=1}^{3} A_{i}=1$,

where $\tau_{1}$ and $\tau_{2}$ are the correlation times and the constant $A_{3}$ has been introduced in order to take into account for the long time limit of the autocorrelation function. At each temperature, the autocorrelation functions have been fitted by means of four free parameters, i.e. the amplitudes $\left(A_{1}\right.$ and $\left.A_{2}\right)$ and characteristic times $\left(\tau_{1}\right.$ and $\left.\tau_{2}\right)$ of the two exponentials. This fitting procedure leads to inconsistent results in terms of temperature dependence of the characteristic times. The amplitude parameters have then been constrained: it has been assumed that the proportionality of the exponential amplitudes (i.e. $A_{1} / A_{2}$ ) was independent of the temperature. Such assumption is justified by the fact that the geometrical properties of the THF dynamics do not evolve with the temperature and that the clathrate structure is identical in the whole temperature range. Thus, for each component of the autocorrelation function, the whole temperature range has been fitted by means of expression (9) via the four parameters $A_{1}, A_{2}, \tau_{1}$ and $\tau_{2}$ with the constraint that the ratio $A_{1} / A_{2}$ is temperature independent. As shown in Fig. 10, an overall good agreement has been obtained. The ratio $A_{1} / A_{2}$ was $1.17,1.12$ and 1.32 for the functions $\langle x(0) . x(t)\rangle,\langle y(0) . y(t)\rangle$ and $\langle z$ $(0) . z(t)>$, respectively; the weight of the two exponential functions are in the same range whatever the molecular axis is. The fitted correlation times are shown in Fig. 11. Whatever is the component of the autocorrelation function, each exponential function possesses the same correlation time and an Arrhenius law $\left(\tau_{i}(T)=\right.$ $\left.\tau_{i}^{0} \exp \left(-E_{i}^{a} / k T\right)\right)$ has been fitted to the thermal variation (by excluding the data obtained at $50 \mathrm{~K}$ ). The fitted prefactors were $\tau_{1}^{0}=0.55 \pm 0.7 \mathrm{ps}$ and $\tau_{2}^{0}=0.22 \pm 0.7 \mathrm{ps}$ respectively for $\tau_{1}(T)$ and $\tau_{2}(T)$. The fitted activation energies were $E_{1}^{a}=0.3 \pm 0.1 \mathrm{~kJ} \cdot \mathrm{mol}^{-1}$ and $E_{2}^{a}=3.0 \pm 0.3 \mathrm{~kJ} \cdot \mathrm{mol}^{-1}$.

Two dynamical processes are clearly identified: (i) the first relaxation process is associated with fast reorientational fluctuations occurring on a sub-picosecond timescale characterized with a small energy barrier and (ii) the second relaxation process is associated with the whole reorientational motions of the THF molecules. The attribution of process (ii) is consolidated with previous analysis of MD simulations [50]: the characteristic time of
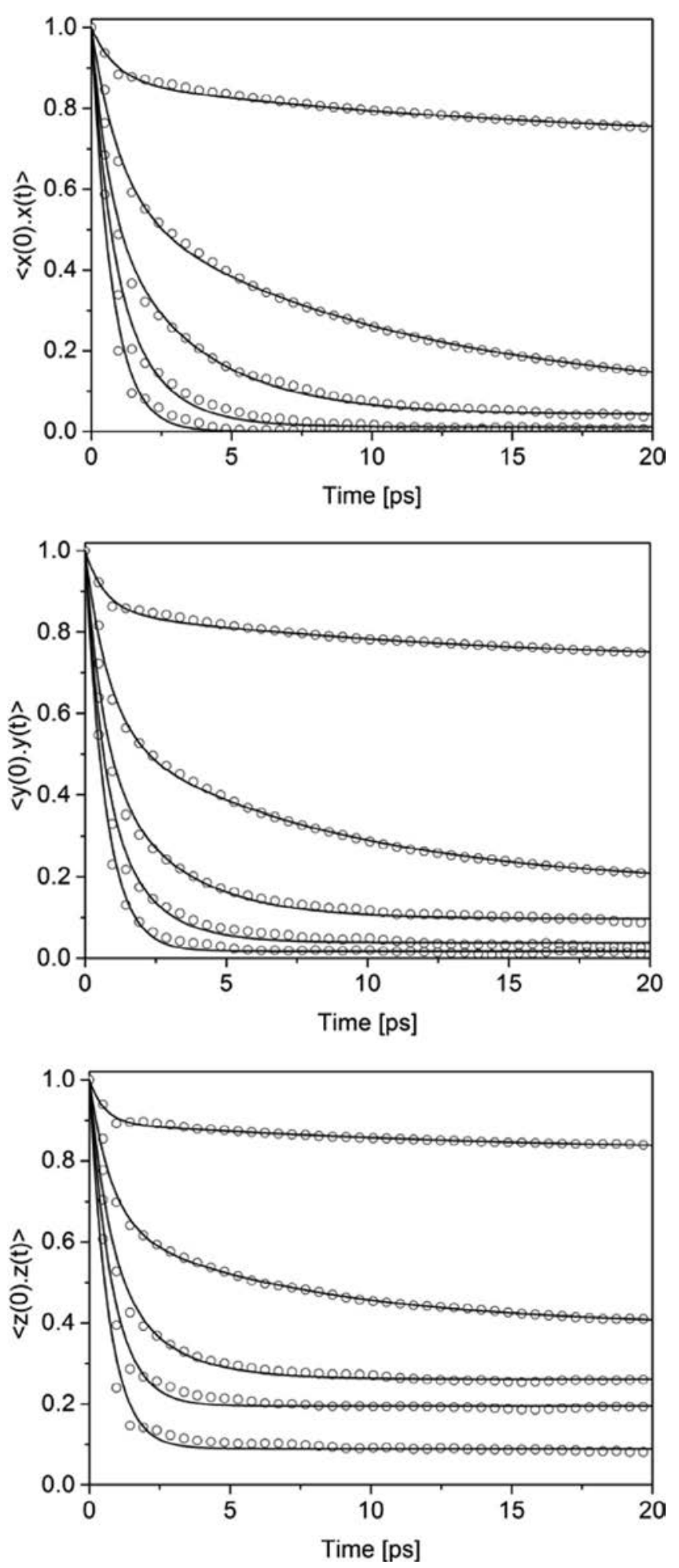

Fig. 10. MD-derived (points) and fitted autocorrelation functions of the THF molecular axis. The temperatures are $50 \mathrm{~K}, 100 \mathrm{~K}, 150 \mathrm{~K}, 200 \mathrm{~K}$ and $270 \mathrm{~K}$ from top to bottom curves. The time sampling has been selected to $0.48 \mathrm{ps}$ in order to minimize the impact of the in-plane excitation.

the dipole-dipole autocorrelation function (analogous to the present autocorrelation $\langle z(0) . z(t)>$ ) is $1.51 \mathrm{ps}$ at $200 \mathrm{~K}$, in good agreement with the correlation time of $1.39 \mathrm{ps}$ at $200 \mathrm{~K}$, calculated with the help of the Arrhenius law of $\tau_{2}(T)$. Moreover, the activation energy of process (ii) is also in agreement with the one determined 

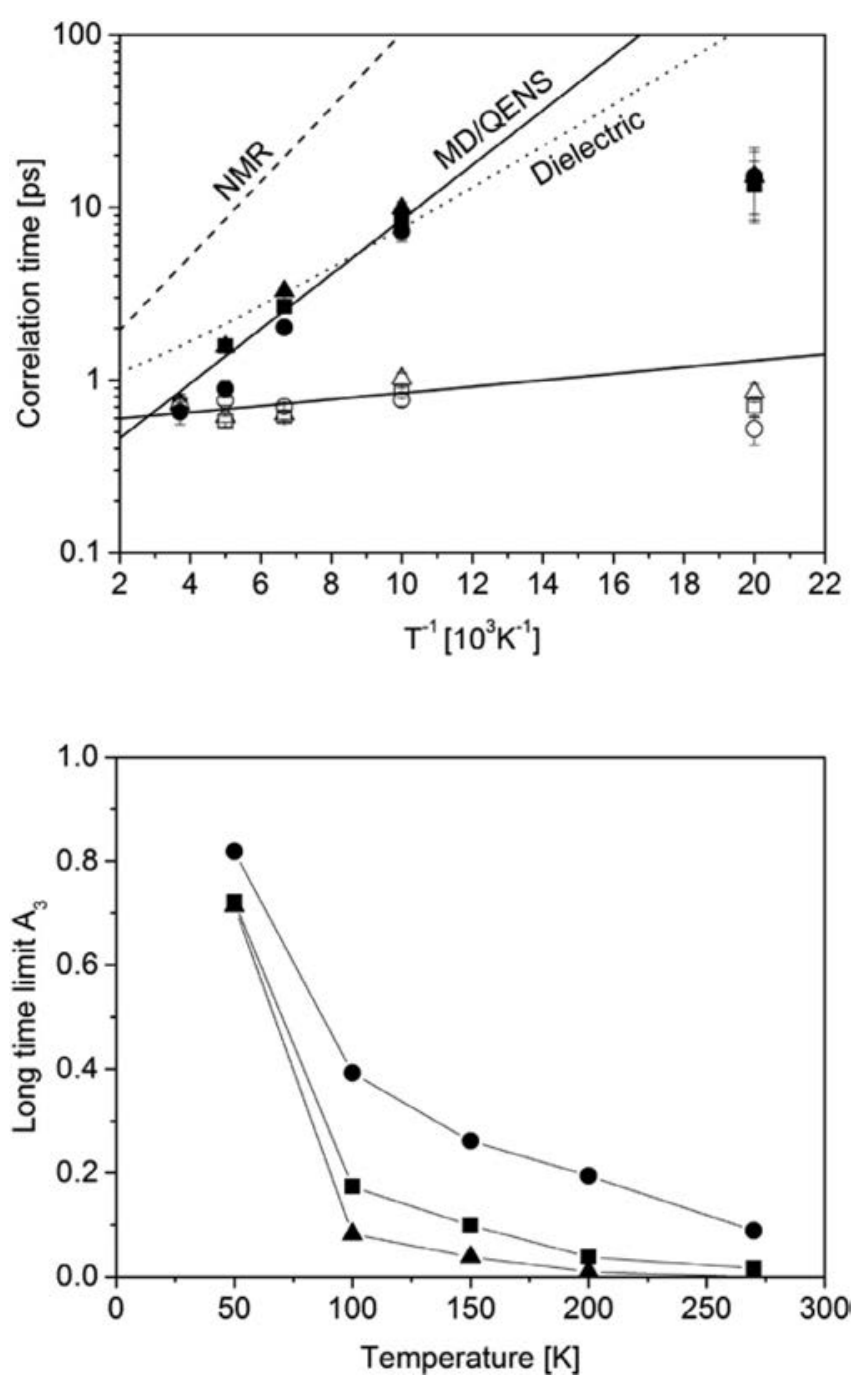

Fig. 11. Temperature dependence of the rotational correlation time (top) and the long time limit (bottom) of the autocorrelation function $\langle x(0) \cdot x(t)\rangle$ (triangles), $<y$ $(0) . y(t)\rangle$ (squares) and $\langle z(0) \cdot z(t)\rangle$ (circles). Top: the open symbols refer to the correlation time $\tau_{1}$, the filled symbols to $\tau_{2}$ and the continuous lines are the fitted Arrhenius laws. The dotted and dashed lines correspond to the Arrhenius behaviour measured by means of dielectric measurements [34] and of solid state ${ }^{2} \mathrm{H}$ NMR [42], respectively.

in previous analyzis of rotational THF motions: $E_{a}$ has been measured ranging from $2.2 \mathrm{~kJ} \cdot \mathrm{mol}^{-1}$ to $3.84 \mathrm{~kJ} \cdot \mathrm{mol}^{-1}$ (determined from spin-lattice relaxation time data in the temperature range 125$243 \mathrm{~K}[39,72]$ ) and up to $4.12 \mathrm{~kJ} \cdot \mathrm{mol}^{-1}$ (determined from stimulated echo data at temperatures below $100 \mathrm{~K}$ [42]) by means of ${ }^{2} \mathrm{H}$ NMR. It has been determined as $2.3 \pm 0.2 \mathrm{~kJ} \cdot \mathrm{mol}^{-1}$ by fitting an Arrhenius law to correlation times measured by dielectric spectroscopy [34]. Fig. 11 includes the temperature dependence determined by means of these two techniques. While the agreement is correct for the activation energies, the MD-derived correlation times are in agreement with dielectric measurements, but nevertheless shorter than NMR results. Differences between dielectric and NMR correlation times have been discussed previously [37$38,42]$. In the present case, it should be noticed that ${ }^{2} \mathrm{H}$ NMR spectra are typical of the so-called rapid regime for $\mathrm{T}>100 \mathrm{~K}$, so that the correlation time is shorter than $10^{-9} \mathrm{~S}$ (with no accurate determination of the correlation time from NMR profile analyzis) [39], in full agreement with the present results. It follows that NMRderived correlation times of Fig. 11 are extrapolated from NMR measurements below $100 \mathrm{~K}$. Moreover, no significant distribution of the correlation time of process (ii) is observed in the present analyzis, except for the one introduced by the fluctuating reorientational process (i). According to ${ }^{2} \mathrm{H}$ NMR analyzis [39-41], no relaxation time distribution is observed above $100 \mathrm{~K}$. Deviation from the exponential relaxation - interpreted with the help of stretched exponential and thus representative of correlation time distribution (see e.g. [73]) - is observed for temperatures below $100 \mathrm{~K}[40,42]$. These ${ }^{2} \mathrm{H}$ NMR analyzes are thus in full agreement with the present study.

Finally, the two reorientational processes (i) and (ii) occur on different timescales with different activation energies, but no anisotropy is observed in terms of characteristic times (i.e. the three molecular axes relax on identical timescale). The anisotropy character of the reorientational process is observed on the temperature dependence of the long time limit (see Fig. 11): the one of the $<z(0)$. $z(t)>$ autocorrelation function is higher than the ones of the two other components. This limit may be associated to the existence of reorientational process occurring on a significantly longer timescale than the MD observation time, as observed by means of ${ }^{2} \mathrm{H}$ NMR $[39,42]$. In other words, on the probed timescale, this limit could be interpreted as a parameter representative of the anisotropic character of the potential experienced by the THF molecules, i.e. of the probability of the preferred orientations in agreement with structural analyzis by means of neutron Powder Diffraction [32] and with ${ }^{2} \mathrm{H}$ NMR data analyzis [39]. By lowering the temperature, this parameter increases while the THF molecule gets stuck in one preferred orientation along the $z$ molecular axis, i.e. the molecular axis containing the guest oxygen atom. In agreement with previous results $[50,74]$, this preferred orientation might thus be associated to the H-bonding between the THF molecules and the water molecules, as revealed by the radial pair distribution function calculated according to the distance between the oxygen atom of the THF molecule and the water hydrogen atoms (Fig. 3).

\section{Summary and concluding remarks}

By combining quasielastic neutron scattering (QENS) experiments and molecular dynamics (MD) simulations, a comprehensive model of the reorientational dynamics of the THF molecule encapsulated in the large cage of the type II clathrate hydrate has been elaborated for temperatures above $100 \mathrm{~K}$. The MD-derived elastic incoherent structure factor (EISF) as well as the MD-derived quasielastic broadening compare with the experimental ones with a good agreement. Spatial and time characteristics of the THF dynamics obtained by means of MD simulations are then consistent with those experimentally determined. Such comparison of experimental and MD-derived neutron scattering laws in the quasielastic region (i.e. for energy smaller than $3 \mathrm{meV}$ and for momentum transfer ranging from $0.5 \AA^{-1}$ to $2.5 \AA^{-1}$ ) leads to the experimental validation of the MD simulations for the first time in the case of the THF clathrate hydrate. The MD trajectories has then been analyzed to develop a model reproducing the complex THF dynamics, in conjunction with known results from dielectric spectroscopy [34], NMR [39,42], neutron diffraction [32] and other MD simulations [50,51].

No long-range translational motion is observed and several processes characterize the reorientations of THF molecule within the cage. To unravel these various components, orientational distribution functions of the THF molecule has been computed in conjunction with autocorrelation functions of the guest molecular axis. A highly damped rotational excitation is observed in the plane of THF molecule with a period of $c a$. 2 ps. In addition to this excitation, orientational relaxations occur with a characteristic time of $0.7 \pm 0.1 \mathrm{ps}$ at $270 \mathrm{~K}$ and are characterized by means of an energy barrier of $3.0 \pm 0.3 \mathrm{~kJ} \cdot \mathrm{mol}^{-1}$. The modeling of the MD trajectories 
leads to a description of the THF dynamics in two types of motions occurring on identical timescale. The THF molecule undergoes rotation about its $C_{2}$ molecular axis. This motion evolves from a continuous rotation at $270 \mathrm{~K}$ to hindered rotations characterized with a modulated orientational distribution function at $50 \mathrm{~K}$. In addition, the $C_{2}$ axis performs anisotropic reorientations by preferentially adopting orientations within the cage. By lowering the temperature to $50 \mathrm{~K}$, the THF molecules get frozen in preferred orientations with respect to the probed timescale. As revealed by the analyzis of selected radial pair distribution function, the preferred orientations adopted by the THF molecules might be associated with the existence of $\mathrm{H}$-bonds between the water molecules and the THF molecules [50], as observed in various clathrate hydrates [74]. The reorientational timescale of the THF molecule (of the order of picosecond) is significantly shorter than the one of water molecules (significantly longer than hundreds of picosecond). This difference of timescale induces a distribution of local environment experienced by the guest molecule and thus the water $\mathrm{H}$-bonds distribution differ from cage to cage on the timescale of MD simulations and of QENS experiments. According to the present analyzis, this various local environment does not induce a distribution of guest relaxation time. The characteristic time associated with the H-bonded host network rearrangement is thus the driven factor of guest preferred orientations within the cage.

\section{Acknowledgements}

The Berlin Neutron Scattering Centre (HZB, Berlin, Germany) is thanked for the provision of beam time for QENS experiments for which the European Commission is thanked for funding under the sixth Framework Programme through the Key Action: Strengthening the European Research Area, Research Infrastructures [Contract No. RII3-CT-2003-505925 (NMI3)]. The CNRS and the HZB are acknowledged for funding EP's PhD work. Finally, this paper falls in the frame of the project HyDfect of the "Agence Nationale de la Recherche" ANR 2011-JS08-002-01.

\section{References}

[1] E.D. Sloan, C.A. Koh, Clathrate Hydrates of Natural Gases, third ed., Taylor \& Francis-CRC Press, Boca Raton, FL, 2008.

[2] E.D. Sloan, Nature 426 (2003) 353-359.

[3] C.A. Koh, E.D. Sloan, AIChE J. 53 (2007) 1636-1643.

[4] A.K. Sum, C.A. Koh, E.D. Sloan, Ind. Eng. Chem. Res. 48 (2009) 7457-7465.

[5] M.R. Walsh, C.A. Koh, E.D. Sloan, A.K. Sum, D.T. Wu, Science 326 (2009) 1095 1098.

[6] J.S. Tse, D.D. Klug, J.Y. Zhao, W. Sturhahn, E.E. Alp, J. Baumert, C. Gutt, M.R. Johnson, W. Press, Nat. Mater. 4 (2005) 917-921.

[7] K. Shin, J. Cha, Y. Seo, H. Lee, Chem. Asian J. 5 (2010) 22-34.

[8] R.M. Rueff, E.D. Sloan, Ind. Eng. Chem. Proc. Des. Dev 24 (1985) 882-885.

[9] I. Cameron, Y.P. Handa, T.H.W. Baker, Can. Geotech. 27 (1990) 255-258.

[10] T.S. Yun, F.M. Francisca, J.C. Santamarina, C. Ruppel, Geophys. Res. Lett. 32 (2005) L10609.

[11] S. Yun, J.C. Santamarina, C.J. Ruppel, Geophys. Res 112 (2007) B04106.

[12] L.J. Florusse, C.J. Peters, J. Schoonman, K.C. Hester, C.A. Koh, S.F. Dec, K.N. Marsh, E.D. Sloan, Science 306 (2004) 469-471.

[13] E. Pefoute, E. Kemner, J.C. Soetens, M. Russina, A. Desmedt, J. Phys. Chem. C 116 (2012) 16823-16829.

[14] Q. Zhang, G.J. Chen, Q. Huang, C.Y. Sun, Z.Q. Guo, Q.L. Ma, J. Chem. Eng. Data 50 (2005) 234-236.

[15] C.Y. Sun, C.F. Ma, G.J. Chen, S.X. Zhang, Fluid Phase Equilib. 261 (2007) 85-91.

[16] A. Delahaye, L. Fournaison, S. Marinhas, I. Chatti, Ind. Eng. Chem. Res. 45 (2006) 391-397.

[17] A.Y. Manakov, S.V. Goryainov, A.V. Kurnosov, A.Y. Likhacheva, Y.A. Dyadin, E.G. Larionov, J. Phys. Chem. B 107 (2003) 7861-7866.
[18] Y. Zhang, P.G. Debenedetti, R.K. Prud'homme, B.A. Pethica, J. Phys. Chem. B 108 (2004) 16717-16722.

[19] E. Tombari, S. Presto, G. Salvetti, G.P. Johari, J. Chem. Phys. 124 (2006) 154507.

[20] S. Gao, W.G. Chapman, W. House, J. Magn. Reson. 197 (2009) 208-212.

[21] F. Lehmkühler, A. Sakko, C. Sternemann, M. Hakala, K. Nygård, C.J. Sahle, S. Galambosi, I. Steinke, S. Tiemeyer, A. Nyrow, T. Buslaps, D. Pontoni, M. Tolan, K. Hämääinen, J. Phys. Chem. Lett. 1 (2010) 2832-2836.

[22] F. Lehmkühler, A. Sakko, I. Steinke, C. Sternemann, M. Hakala, C.J. Sahle, T Buslaps, L. Simonelli, S. Galambosi, M. Paulus, T. Pylkkänen, M. Tolan, K. Hämäläinen, J. Phys. Chem. C 115 (2011) 21009-21015.

[23] J.Y. Wu, L.J. Chen, Y.P. Chen, S.T. Lin, J. Phys. Chem. C 119 (2015) 1400-1409. [24] G.P. Johari, H.A.M. Chew, Nature 303 (1983) 604-605.

[25] G.P. Johari, H.A.M. Chew, Philos. Mag. B 49 (1984) 281-294.

[26] C.A. Tulk, D.D. Klug, J.A. Ripmeester, J. Phys. Chem. A 102 (1996) 8734-8739.

[27] S. Subramanian, E.D. Sloan, J. Phys. Chem. B 106 (2002) 4348-4355.

[28] P.S.R. Prasad, K. Shiva Prasad, N.K. Thakur, Spectrochim. Acta Part A 68 (2007) $1096-1100$.

[29] D.D. Klug, E. Whalley, Can. J. Chem. 51 (1973) 4062-4071.

[30] H.H. Richardson, P.J. Wooldridge, J.P. Devlin, J. Chem. Phys. 83 (1985) 43874394.

[31] T.C. Mak, R.K. McMullan, J. Chem. Phys. 42 (1965) 2732-2737.

[32] O. Yamamuro, T. Matsuo, H. Suga, W.I.F. David, R.M. Ibberson, A.J. Leadbetter, Phys. B Condens. Matter 213-214 (1995) 405-407.

[33] C.Y. Jones, S.L. Marshall, B.C. Chakoumakos, C.J. Rawn, Y. Ishii, J. Phys. Chem. B 107 (2003) 6026-6031.

[34] M. Davies, K. Williams, Trans. Faraday Soc. 64 (1968) 529.

[35] D.W. Davidson, in: F. Franks (Ed.), Clathrate Hydrates in Water-A Comprehensive Treatise, Plenum Press, New York, 1973.

[36] O. Yamamuro, T. Matsuo, H. Suga, J. Inclusion Phenom. 8 (1990) 33-44.

[37] S.K. Garg, D.W. Davidson, J.A. Ripmeester, J. Magn. Reson. 15 (1974) 295-309.

[38] D.W. Davidson, S.K. Garg, J.A. Ripmeester, J. Magn. Reson. 31 (1978) 399-410.

[39] M. Bach-Vergés, S.J. Kitchin, K.D.M. Harris, M. Zugic, C.A. Koh, J. Phys. Chem. B 105 (2001) 2699-2706.

[40] T.M. Kirschgen, M.D. Zeidler, B. Geil, F.A. Fujara, Phys. Chem. Chem. Phys. 5 (2003) 5243-5246.

[41] T.M. Kirschgen, M.D. Zeidler, B. Geil, F.A. Fujara, Phys. Chem. Chem. Phys. 5 (2003) 5247-5252.

[42] A. Nowaczyk, B. Geil, S. Schildmann, R. Böhmer, Phys. Rev. B 80 (2009) 144303.

[43] A. Desmedt, F. Stallmach, R.E. Lechner, D. Cavagnat, J.C. Lassegues, F. Guillaume, J. Grondin, M.A. Gonzalez, J. Chem. Phys. 121 (2004) 11916-11926.

[44] A. Desmedt, R.E. Lechner, J.C. Lassegues, F. Guillaume, D. Cavagnat, J. Grondin, Solid State Ionics 252 (2013) 19-25.

[45] L. Bedouret, P. Judeinstein, J. Ollivier, J. Combet, A. Desmedt, J. Phys. Chem. B 118 (2014) 13357-13364.

[46] A. Desmedt, L. Martin-Gondre, T.T. Nguyen, C. Pétuya, L. Barandiaran, O. Babot, T. Toupance, R.G. Grim, A.K. Sum, J. Phys. Chem. C 119 (2015) 8904-8911.

[47] R.J. Hayward, K.J. Packer, Mol. Phys. 25 (1973) 1443-1450.

[48] C. Albayrak, M.D. Zeidler, R. Kuchler, O. Kanert, Ber. Bunsen-Ges. Phys. Chem. 93 (1989) 1119-1122.

[49] D.M. Jacobs, M.D. Zeidler, O. Kanert, J. Phys. Chem. A 101 (1997) 5241-5249.

[50] S. Alavi, R. Susilo, J.A. Ripmeester, J. Chem. Phys. 130 (2009) 174501.

[51] S. Alavi, J.A. Ripmeester, J. Chem. Phys. 137 (2012) 054712.

[52] M.J. Shultz, T.H. Vu, J. Phys. Chem. B 119 (2015) 9167-9172.

[53] A. Desmedt, J.C. Soetens, M. Prager, M. Russina, J. Ollivier, J. Phys. Chem. C 115 (2011) 12689-12701.

[54] A. Desmedt, L. Bedouret, E. Pefoute, M. Pouvreau, S. Say-Liang-Fat, M. Alvarez, Eur. Phys. J. Spec. Top. 213 (2012) 103-127.

[55] J. Ollivier, H. Mutka, J. Phys. Soc. Jpn. 80 (2011), SB003.

[56] A. Desmedt, Contact A.D. for further details about the software, 2016.

[57] H.J.C. Berendsen, J.R. Grigera, T.P. Straatsma, J. Phys. Chem. 91 (1987) 62696271.

[58] Gaussian 09, Revision A.02, M. J. Frisch, G. W. Trucks, H. B. Schlegel, G. E. Scuseria, M. A. Robb, J. R. Cheeseman, G. Scalmani, V. Barone, B. Mennucci, G. A. Petersson, H. Nakatsuji, M. Caricato, X. Li, H. P. Hratchian, A. F. Izmaylov, J. Bloino, G. Zheng, J. L. Sonnenberg, M. Hada, M. Ehara, K. Toyota, R. Fukuda, J. Hasegawa, M. Ishida, T. Nakajima, Y. Honda, O. Kitao, H. Nakai, T. Vreven, J. A. Montgomery, Jr., J. E. Peralta, F. Ogliaro, M. Bearpark, J. J. Heyd, E. Brothers, K. N. Kudin, V. N. Staroverov, R. Kobayashi, J. Normand, K. Raghavachari, A. Rendell, J. C. Burant, S. S. Iyengar, J. Tomasi, M. Cossi, N. Rega, J. M. Millam, M. Klene, J. E. Knox, J. B. Cross, V. Bakken, C. Adamo, J. Jaramillo, R. Gomperts, R. E. Stratmann, O. Yazyev, A. J. Austin, R. Cammi, C. Pomelli, J. W. Ochterski, R. L. Martin, K. Morokuma, V. G. Zakrzewski, G. A. Voth, P. Salvador, J. J. Dannenberg, S. Dapprich, A. D. Daniels, Ö. Farkas, J. B. Foresman, J. V. Ortiz, J. Cioslowski, and D. J. Fox, Gaussian, Inc., Wallingford CT, 2009.

[59] A.D. Becke, J. Chem. Phys. 98 (1993) 5648-5652.

[60] C.M. Breneman, K.B. Wiberg, J. Comput. Chem. 11 (1990) 361-373.

[61] W.D. Cornell, P. Cieplak, C.I. Bayly, I.R. Gould, K.M. Merz, D.M. Ferguson, D.C. Spellmeyer, T. Fox, J.W. Caldwell, P.A. Kollman, J. Am. Chem. Soc. 117 (1995) 5179-5197.

[62] J.C. Soetens, PhD. Thesis, Université Henri Poincaré-Nancy I, 1996.

[63] J.C. Soetens, A. Desmedt, F. Guillaume, K.D.M. Harris, Chem. Phys. 261 (2000) $125-135$

[64] A. Desmedt, J.C. Soetens, F. Guillaume, R.E. Lechner, A.J. Dianoux, Appl. Phys. A 74 (2002) 1357-1359.

[65] J.C. Soetens, W.R. Rice, G. Brunaud, A. Desmedt, F. Guillaume, Chem. Phys. 292 (2003) 201-216. 
[66] S.A. Nosé, Mol. Phys. 52 (1984) 255-268.

[67] H.C. Andersen, J. Chem. Phys. 72 (1980) 2384-2393.

[68] M.P. Allen, D.J. Tildesley, Computer Simulations of Liquids, Oxford Science Publications, 1987.

[69] M. Bée, Quasielastic Neutron Scattering, Adam Hilger, Bristol, 1988.

[70] T. Springer, R.E. Lechner, Diffusion in Condensed Matter, 2nd ed., P. Heitjans and J. Kärger Eds., Springerverlag: Berlin \& Heidelberg, 2005, Vol. 1, p.93.

[71] T. Róg, K. Murzyn, K. Binsen, G.R. Kneller, J. Comp. Chem. 24 (2003) 657-667.
[72] D.W. Davidson, J.A. Ripmeester, NMR, NQR and dielectric properties of clathrates, in: J.L. Atwood, J.E.D. Davies, D.D. MacNicol (Eds.), Inclusion Compounds, vol. 3, Academic Press, Orlando, 1984.

[73] G. Arialdi, K. Karatasos, J.P. Ryckaert, V. Arrighi, F. Saggio, A. Triolo, A. Desmedt, J. Pieper, R.E. Lechner, Macromolecules 36 (2003) 8864-8875.

[74] V. Buch, J.P. Devlin, I.A. Monreal, B. Jagoda-Cwiklik, N. Uras-Aytemiz, L. Cwiklik, Phys. Chem. Chem. Phys. 11 (2009) 10245. 